\title{
Circuit Design for Thermal Compensation of Avalanche Photodiode
}

\author{
Muhammad Tajammal Chughtai \\ Department of Electrical Engineering, College of Engineering \\ University of Hail, Hail, Saudi Arabia \\ mt.chughtai@uoh.edu.sa
}

\begin{abstract}
It has been observed that the breakdown voltage of the avalanche photodiode (APD) changes with the change in the ambient temperature. This situation may result in a poorer signal to noise ratio and sometimes to permanent damage to the APD. In order to overcome these problems, various considerations may be taken into account, including maintaining the temperature of the APD permanently or the design of a bias system, which would be self-adjusting according to any changes in the temperature. The latter technique was adopted and the design of a bias supply is presented in this article.
\end{abstract}

Keywords-APD; thermal adjustment; optoelectronics

\section{INTRODUCTION}

Avalanche photodiodes (APDs) are a good solution to the situations where very week signal is expected [1]. Over the years, researchers have developed various techniques in modeling and manufacture of APDs. Authors in [2] suggested a new technique towards etching of epitaxial layer in an APD. Authors in [3] developed a technique for temperature tracking using temperature sensors for $\mathrm{Si}$ photodiode. Authors in [4] studied and reported the dependence of APDs' temporal stability on a range of temperature variations. Authors in [5] also presented a study on photodiodes' dependence on temperature variations. Diverse approaches have been adapted by researchers in the field of optoelectronics to come up with a range of promising solutions in relation to photodiodes. Embedded version of photodiodes in technical textiles has been reported in [6]. APDs have been known for their sensitivity towards changes in the ambient temperature, their operating point keeps shifting i.e. with increase in the temperature their operating point shifts away from the original breakdown point, which results in less sensitivity and reduced amplitude of the signal output. On the other hand, a drop in the temperature will shift the breakdown point to a lesser value, which may result in increased sensitivity and enhanced multiplication noise, which may eventually result into a permanent damage to the APD [7]. A study on APD in relation to its performance in extreme variations of temperature environment was conducted in [8]. The remedy to these problems is thought to be in the form of a temperature compensated bias system, which is capable of keeping track of any changes in the ambient temperature and hence providing a higher safety to the APD. A self calibrating photo detector at room temperature while operating it in dual mode has been reported in [9]. Authors in [10] discussed on the variety of parameters related to PIN photodiodes from the point of view of its manufacturing and physics involved. Authors in [11] developed a 3D structure version of InP/InGaP APD and claimed low noise factor figures.

\section{VARIOUS SUGGESTED METHODS TO MAINTAIN THE MULTIPLICATION FACTOR}

To keep the multiplication factor constant for a constant level of input signal it is necessary for the reverse bias voltage fed to the APD to track any changes in the temperature otherwise, changes in the temperature of the APD may cause the breakdown voltage of the APD to excess or the APD may give a signal of lower amplitude than desired. If the applied voltage exceeds the breakdown voltage (as a result of decrease in temperature), a permanent damage to the APD may occur. Additionally, a rise in temperature will shift the breakdown point, leading to a signal of a lower than desired amplitude. Three most common solutions to this problem are: (i) constant current operation, (ii) maintenance of the temperature of the APD at a constant level, and (iii) bias the APD using a temperature compensated power supply. A constant current power supply scheme can be seen in [12] along with the derived formulas to compensate the temperature variations and hence devised methods to stabilize the operating the operation of an APD. Two types of temperature coefficients $\gamma$ and $\beta$ were used, where $\gamma$ represents the change in multiplication factor due to temperature variation while the APD is biased under constant voltage condition and $\beta$ describes the change in avalanche voltage with respect to temperature when the APD is operating under constant multiplication factor. Multiplication factor $(M)$ is related to ionization rate $(\alpha)$ as:

$$
1-M^{-1}=\int \alpha d x
$$

The solution of this integral has been suggested in terms of width of avalanche region in [15]. One method of temperature compensation can be achieved by ensuring that the voltage of the APD tracks any temperature change by using another APD to produce a reference voltage signal [13]. The second method mentioned above, causes condensation on the APD window and hence a poorer response and need to clean the window. The third method of stabilizer and APD is to design a circuit using temperature sensing components, as shown in Figure 1.

Corresponding author: Muhammad Tajammal Chughtai 


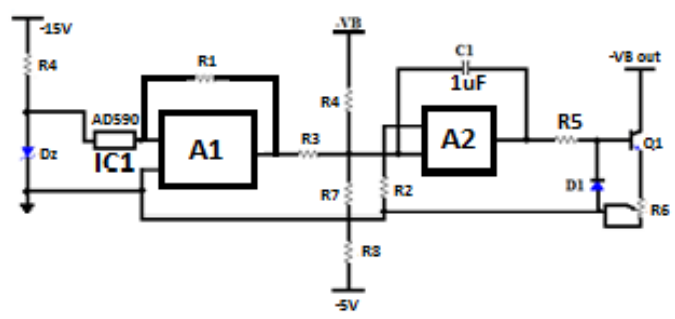

Fig. 1. Schematic of temperature compensated bias supply for APD.

Usually, temperature sensing components produce a current signal which varies with any change in temperature. This output current may be converted to a voltage using an operational amplifier, configured as a current to voltage converter. The output of this op-amp can then be fed to a summing amplifier in a non-inverting configuration, thereby summing the voltage with the negative value from a conventional high voltage power supply and a reference voltage. The resultant output is used to control the base current of the bias control transistor.

\section{DESIGN CONSIDERATIONS}

The APD used for this design study was an NEC model NDL-1202, the temperature coefficient $\beta$ for this particular APD is $1 \times 10^{-1} \%{ }^{\circ} \mathrm{C}$ [14]. In order to convert this temperature coefficient into a voltage variation $\left(\right.$ Volts $\left./{ }^{\circ} \mathrm{C}\right)(1)$ is used:

$$
\frac{\beta}{V_{B R}}=1 \times 10^{-1} \times 100
$$

where $\beta$ is temperature coefficient of the APD in Volts $/{ }^{\circ} \mathrm{C}$ and VBR is the reverse breakdown voltage of the APD. The value of VBR for this case is $213.5 \mathrm{~V}$, so that (1) yielded [7]:

$$
\beta=213.5 \mathrm{mV} /{ }^{\circ} \mathrm{C} .
$$

The circuit for a temperature compensated power supply for an APD is illustrated in Figure 1, a step by step design and analysis of the circuit follows.

A. Step-1

A constant voltage reference of $-6.8 \mathrm{~V}$ was obtained using a precision reference diode package LM329. This constant reference voltage was then supplied to the temperature sensor IC AD590, which has a temperature coefficient of $1 \mu \mathrm{A} /{ }^{\circ} \mathrm{K}$.

\section{B. Step-2}

Consider the combination of the temperature sensor ICI and the operational amplifier A1. The total current generated by IC1 for a rise of $T$ Kelvin degrees in the temperature will be $T \times 10^{-6}$ A. Hence, the current $I$ supplied by the temperature sensor for a rise of $T$ will be:

$$
I=T \times 10^{-6 \mathrm{~A} / \mathrm{K}}
$$

Therefore, the voltage at the output of the op-amp A1 with feedback resister $R_{1}$ can be expressed as:

$T \times 10^{-6}=V_{1} / R_{1}$.

Thus, the voltage change $\mathrm{V}_{1}$ becomes:

$$
V_{1}=R_{1} \times T \times 10^{-6} \mathrm{~V}
$$

\section{Step-3}

The operational amplifier A2 was used in a summing amplifier configuration so that it added all three voltages applied at its inputs to produce the output $V o$. This output $V o$ was supplied at the base of an NPN transistor Q1, through a resistor. Transistor Q1 was configured so that the smaller the voltage signal at its base, the lower was the conduction between its collector and emitter. When conducting, the transistor produced a flow of current between its collector and emitter of Q1. In turn, this caused the $+\mathrm{HV}$ point to float, hence, a variation in the HV point at the output could be observed. Kirchhoff's law states that, at any point in an electrical circuit, the algebraic sum of the currents meeting at that point is zero. Therefore, the current equation according to Kirchhoff's law at the input of A2 can be written as.

$$
\frac{V_{r e f}}{R_{2}}+\frac{V_{B}}{R_{4}}+\frac{V_{1}}{R_{3}}=0
$$
have:

However, substituting the value of $V_{l}$ from (4) in (5) we

$$
\frac{V_{r e f}}{R_{2}}+\frac{V_{B}}{R_{4}}+\frac{T \times R_{1} \times 10^{-6}}{R_{3}}=0
$$

Differentiating (6), with respect to $T$ leads to:

$\frac{d V_{B}}{d T}=-\frac{R_{1} \times R_{4} \times 10^{-6}}{R_{3}}$

Putting up $\frac{d V_{B}}{d T}=\beta,(7)$ becomes:

$$
\beta=-\frac{R_{1} \times R_{4} \times 10^{-6}}{R_{3}}
$$

which gives the value for the temperature coefficient $\beta$ of the APD. Substituting this value into (5) gives:

$$
V_{B}=-\frac{V_{r e f} \times R_{4}}{R_{2}}+T \times \beta
$$

The values for the resistors $R_{2}, R_{3}$ and $R_{4}$ can be calculated by solving (5), (8) and (9) simultaneously.

\section{PRACTICAL IMPLEMENTATION}

From the data provided with the APD, the reverse breakdown voltage of the particular APD under consideration was found to be $213.50 \mathrm{~V}$ and, according to this value, the temperature coefficient was $213.50 \mathrm{mV} / \mathrm{K}$. In order to calculate the appropriate values of the various resistors to be used in the circuit, this voltage value must be substituted into (2) using $R_{l}=20 \mathrm{k} \Omega$. This yields:

$$
-213.5 \times 10^{-3}=\frac{20 k \times R_{4}}{R_{3}} \times 10^{-6}
$$

Thus,

$$
\frac{R_{4}}{R_{3}}=\frac{213.5}{20}=10.675
$$

leading to the value $R_{4}=11 R_{3}$. Since it was already required that the APD should operate at a temperature of $300 \mathrm{~K}$ (i.e, approximating to a normal room temperature), this implied that the following values were already fixed: 


\section{$T=300^{\circ} \mathrm{K}, \beta=-213.5 \mathrm{mV} /{ }^{\circ} \mathrm{K}, R_{l}=20 \times 10^{3} \Omega$}

Inserting these values in (9) led to a value for the reversed breakdown voltage:

$$
V_{B}=-V_{\text {ref }} \frac{R_{4}}{R_{2}}-64.05
$$

Furthermore, it was required to operate the APD at a reverse breakdown voltage value of $V_{B}=-212.5 \mathrm{~V}$, so that the avalanche noise was at optimum level. Therefore

$$
-212.5+64.05=-V_{\text {ref }} \frac{R_{4}}{R_{2}}
$$

but

$\mathrm{V}_{\text {ref }}=5$ Volts

$\therefore \frac{R_{4}}{R_{2}}=29.69$

Assuming that $R_{2}=100 \mathrm{k} \Omega,(10)$ yields:

$R_{4}=1000 \mathrm{k}(29.69)=2969 \mathrm{k} \approx 3 \mathrm{M} \Omega$

$R_{3}=\frac{3 \mathrm{M}}{10.675}=281 \mathrm{k} \Omega$

Inserting these design values for $R_{1}, R_{2}, R_{3}$ and $R_{4}$ into the circuit design, satisfactory performance of the temperature compensated power supply for the APD was obtained. However, to set the value of the temperature coefficient precisely, it was decided to insert a variable resistor instead of a fixed value for $R_{2}$. This could then be adjusted until the required output value for bias voltage was obtained. Apart from the present application, the implementation of calculations may also be employed to temperature sensitive environment and systems such as shop-floor gauging, altimeter temperature correction and other sensor temperature compensations.

\section{RESULTS AND CONCLUSION}

Application of the circuit under consideration was applied to bias an APD which have been used at the receiving end of a laser Doppler anemometer (LDA) system in back scatter mode. Since in backscatter mode the signal intensity is far lower compared to forward scatter, the necessity of using an APD arose. Regarding APD's performance with respect to thermal changes the application of such circuit became compulsory so that reasonable amplitude of the signal could be maintained. The circuit for temperature compensation of the APD was implemented and assembled on a double-sided printed circuit board, according to the design values of the components calculated above (Figure 2). Tests were carried out to the designed circuit by applying different load conditions to HV power supply. For such cases it is very common to pick up noise by oscilloscope probes. In this case, ripple was also observed, an average pk-pk value of $15.35 \mathrm{mV}$ and an rms value of $3.30 \mathrm{mV}$ at a frequency of $50 \mathrm{~Hz}$ were observed. Tests on HV supply were carried out by employing a range of loads having values $\infty, 1 \mathrm{M}, 10 \mathrm{k}$ and $47 \mathrm{k} \Omega$. Results in terms of noise frequency in $\mathrm{Hz}$ and ac noise level were collected and are presented in Table I. In order to measure the variation in the circuit performance for changes in the ambient temperature, the temperature sensing semi-conductor element and a thermocouple were both mounted on the same heat sink.

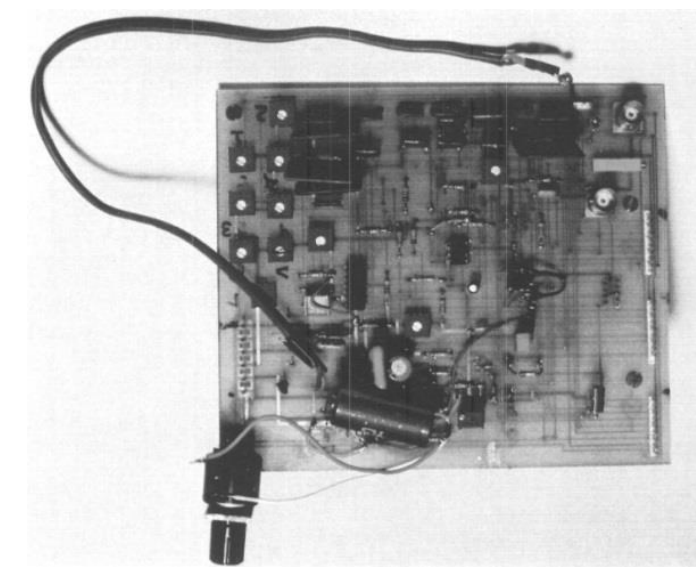

Fig. 2. PCB sharing thermal compensation circuit.

TABLE I. LOAD RESULTS

\begin{tabular}{|c|c|c|}
\hline Load resistance $(\boldsymbol{\Omega})$ & Noise $(\mathbf{H z})$ & ac noise level $\left(\mathbf{V}_{\mathbf{p}-\mathbf{p}}\right)$ \\
\hline$\infty$ & $192 \mathrm{k}$ & 0.003 \\
\hline $1 \mathrm{M}$ & 50 & -0.034 \\
\hline $10 \mathrm{k}$ & 100 & 0.195 \\
\hline $47 \mathrm{k}$ & 100 & 0.462 \\
\hline
\end{tabular}

The thermocouple was used to monitor the temperature variations directly. The heat sink itself was coiled around by a $1 \mathrm{~m}$ long high resistance Eureka wire for heating purposes. The two ends of this wire were attached to a variable current power supply, so that it could be heated, thereby raising the temperature of the heat sink, and varying the amplitude of the signal from the temperature sensing element to the high voltage (HV) circuit. The variation in the output voltage from the circuit was observed. The output of the high voltage power supply was attached to a voltage ratio divider unit, which helped reduce the high voltage to an appropriate level which could be supplied to an analogue to digital converter card fitted inside a personal computer. The arrangement used can be seen in [5]. A number of observations were taken for different heat sink temperatures, with data being collected in the temperature range of $20-77^{\circ} \mathrm{C}$. The results shown in Figure 3 confirm that the output signal of the HV power supply circuit follows a reasonably linear behavior in accordance with temperature variations.

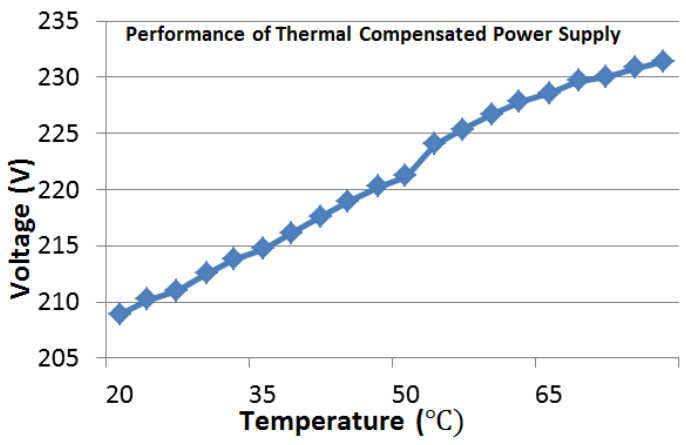

Fig. 3. Performance profile of designed circuit.

Such deviations from linear behavior may be caused by the non-linear behavior of components used to construct the 
temperature compensation circuit. The design methodology and calculations mentioned above are versatile and can easily be used in applications where variation of temperature becomes problematic to the operation of a system. This may be carried out by considering appropriate values of temperature coefficients and other discrete component values depending upon power requirement.

\section{REFERENCES}

[1] J. W. Gardener, Micro Sensors: Principles and Applications, Jonn Wiley \& Sons, 1995

[2] A. K. Budtolae, T. N. Grishina, P. E. Khakuashev I. V. Chinareva, "Formation of guard ring of avalanche photodiode based on the InGaAs/InPheterostructure", Journal of Communications Technology and Electronics, Vol. 62, No. 9, pp. 1078-1082, 2017

[3] E. Borreguero, C. K. Tang, J. Gran, A. P. Aglio J. C. Acosta, "Preliminary results of feasibility of self-calibration of silicon pn photodiodes at room temperature using temperature sensors", Optica Pura y Aplicada, Vol. 51, pp. 1-8, 2018

[4] S. Abdullah, C. H. Tan, X. Zhou, S. Zhang, L. Pinel, J. S. Ng, "Investigation of temperature and temporal stability of $\mathrm{AlGaAsSb}$ avalanche photodiodes", Optics Express, Vol. 25, No. 26, pp. 3361033616, 2017

[5] M. T. Chughtai, "Temperature Compensated Bias Supply Circuit for Photodiodes", Przeglad Elektrotechniczny, Vol. 94, No. 10, pp. 207-209, 2018

[6] A. Satharasinghe, T. Hughes-Riley, T. Dias, "Photodiodes embedded within electronic textiles", Scientific Reports, Vol. 8, No. 1, pp. 1-13, 2018

[7] B. Woodward, A. Oliviero, Cabling: The Complete Guide to Copper and Fiber-Optic Networking, Sybex, 2009

[8] T. Y. Chuan, R. Chandrasekara, C. Cheng, A. Ling, "Silicon avalanche photodiode operation and lifetime analysis for small satellites", Optics Express, Vol. 21, pp. 16946-16954, 2013

[9] M. U. Nordsveen, C. K. Tang, J. Gran, "Demonstration of a dual-mode $\mathrm{Si}$ detector as a self-calibratingdevice at roomtemperature", Optics Express, Vol. 25, pp. 8459-8469, 2017

[10] E. Fossum, R. Hondongwa, B. Donald, "A Review of the Pinned Photodiode for CCD and CMOS Image Sensors", IEEE Journal of the Electron Devices Society, Vol. 2, No. 3, pp. 33-43, 2014

[11] Z. Wu, J. Guo, Y. Li, Y. Zhao, "Low-Noise 3-D Avalanche Photodiodes", IEEE Photonics Journal, Vol. 8, No. 4, pp. 1-10, 2016

[12] K. Nishida, M. Nakajima, "Temperature dependence and stabilization of avalanche photodiodes", Review of Scientific Instruments, Vol. 43, No. 9, pp. 1345-1350, 1972

[13] D. Renker, "Properties of avalanche photodiodes for applications in high energy physics, astrophysics and medical imaging", Nuclear Instruments and Methods in Physics Research Section A: Accelerators, Spectrometers, Detectors and Associated Equipment, Vol. 486, No. 1-2, pp. 164-169, 1974

[14] K. Kiya, T. Hayashi, "Direct current method for extremely weak optical signal detection using avalanche photodiodes", Journal of Spectroscopic Society of Japan, Vol. 33, No. 2, pp. 78-85, 1984

[15] R. Hall, "Temperature coefficient of the breakdown voltage of Silicon PN junction", International Journal of Electronics, Vol. 22, No. 6, pp. 513-519, 1967 